\title{
Comparative investigation of antioxidant activity of human serum blood by amperometric, voltammetric and chemiluminescent methods
}

\author{
Evgenii Plotnikov ${ }^{1}$, Elena Korotkova ${ }^{1}$, Olesya Voronova ${ }^{1}$, Natalia Sazhina ${ }^{2}$, Ekatherina Petrova ${ }^{1}$, \\ Anton Artamonov ${ }^{3}$, Ludmila Chernyavskaya ${ }^{4}$, Elena Dorozhko ${ }^{1}$
}

\author{
${ }^{1}$ Tomsk Polytechnic University, Tomsk, Russia \\ ${ }^{2}$ Emanuel Institute of Biochemical Physics, Moscow, Russia \\ ${ }^{3}$ Flamena Itd, Moscow, Russia \\ ${ }^{4}$ Siberian State Medical University, Tomsk, Russia
}

Submitted: 28 July 2014

Accepted: 29 October 2014

Arch Med Sci 2016; 12, 5: 1071-1076

DOI: $10.5114 /$ aoms.2015.50234

Copyright $\odot 2015$ Termedia \& Banach

\section{Corresponding author:} Evgenii Plotnikov Tomsk Polytechnic University Lenin av., 30 634050 Tomsk, Russia Phone: 0079528861086 E-mail: plotnikov.e@mail.ru

\begin{abstract}
Introduction: A blood test can provide important information about the functional state of the antioxidant system. Malfunction of this system increases the concentration of free radicals and can cause oxidative stress. A difficulty in assessing oxidative stress is the lack of a universal method for determining the antioxidant activity (AOA) of blood components, because of their different nature.

Material and methods: The objects of investigation were sera of 30 male patients with a diagnosis of alcohol dependence syndrome and healthy donors. Comparative investigation of total antioxidant activity (TAA) of human serum blood was carried out by voltammetric (VA), amperometric (AM) and chemiluminescent $(\mathrm{HL})$ methods.

Results: All applied methods revealed that serum TAA of the patients with alcoholism is lower than TAA of healthy donors (control group); according to amperometric method the average value of serum TAA was $850 \pm 210$ $\mathrm{nA} \times \mathrm{s}$, and $660 \pm 150 \mathrm{nA} \times \mathrm{s}$ for healthy donors and alcoholics respectively $(p<0.05)$. Similar trend was revealed by chemiluminescence and voltammetry methods. The results confirm that thiol compounds make a significant contribution to the antioxidant activity of serum. The average thiol concentrations were $0.94 \pm 0.34 \mathrm{mmol} / /$ and $1.21 \pm 0.36 \mathrm{mmol} / \mathrm{l}(p<0.05)$ for alcoholics and healthy donors respectively. Decreasing thiol concentration in blood of alcoholics leads to depletion of antioxidant systems of blood. However, the differences between the results of AM, VA and HL methods were significant, because they reflected different aspects of antioxidant activity. Conclusions: For objective assessment of antioxidant activity of biological objects, we suggest using methods based on different model systems.
\end{abstract}

Key words: total antioxidant activity of blood, alcoholism.

\section{Introduction}

A blood test provides comprehensive information about inner conditions and processes. One important parameter is the functional state of the antioxidant system. Malfunction of this system increases the concentration of free radicals (superoxide anion radical and hydroperoxide radical, hydroxyl radical, etc.), the excess of which causes oxidative stress 
and takes part in the pathogenesis of a number of diseases [1-3]. In turn, diseases, environmental factors, and stress lead to the fact that the natural antioxidant system of the body is not able to effectively neutralize radicals, which causes more severe oxidative stress. However, various biological factors, as well as different methods of analysis, result in a large variation in detected blood parameters of the antioxidant system. A difficulty in assessing oxidative stress is the lack of a universal method for determining the antioxidant activity of blood components, because of their different nature. A review [4] showed a low correlation between the most well-known methods for assessing antioxidant activity, which allows us to speak only about the qualitative characteristics of the analysis results.

The aim of this work was to perform a comparative evaluation of the total antioxidant activity (TAA) of human serum blood in healthy conditions and in pathology (alcohol dependence syndrome) by amperometric, chemiluminescence and voltammetric methods. Alcoholism, in general, is characterized by mental and physical dependence on ethanol, which is associated with changes in biochemical parameters in the blood of the patient. The total concentration of thiol compounds was determined in the same samples, as an additional parameter, which also describes the state of the antioxidant system.

\section{Material and methods}

The object of investigation was the serum of 30 male patients with a diagnosis of alcohol dependence syndrome. All patients were under medical care in Tomsk regional psychiatric hospital. Blood serum of healthy donors was used as the control group.

\section{Blood sample pretreatment}

Venous blood, collected fasting, was placed in a glass centrifuge tube without anticoagulant and left at room temperature for 30 min to allow formation of the clot, which was immediately removed. The tubes were centrifuged in a tabletop centrifuge for $10 \mathrm{~min}$ at a rotation speed of $2000 \mathrm{rpm}$. The centrifugate was sampled in the volume of $1.0-1.5 \mathrm{ml}$ for further study.

\section{Chemiluminescence method $(\mathrm{CL})$}

The oxidation system "hemoglobin - hydrogen peroxide - luminol" was used for chemiluminescent determination of TAA of blood serum. The distinguishing feature of this system from other oxidation systems is that the radicals, formed therein, can initiate the same free radical reaction in vivo [5]. Interaction of hydrogen peroxide $\left(\mathrm{H}_{2} \mathrm{O}_{2}\right)$ with me$\mathrm{tHb}$ (methemoglobin) is accompanied by the destruction of heme with the appearance of iron ions, which are involved in the formation of $\mathrm{OH}^{*}$. Furthermore, this interaction forms ferryl-radicals $\left(\mathrm{Hb}\left({ }^{+}\right)-\mathrm{Fe}^{4+}=\mathrm{O}\right)$. Formed radicals initiate the oxidation of luminol. As a result of this oxidation process, luminol endoperoxide $\left(\mathrm{LO}_{2}{ }^{2-}\right)$ and then 3-aminophthalate dianion in the excited state $\left(\mathrm{AP}^{2-}\right)$ are formed. Transition of these ions to the ground state cause emission of photons with a wavelength of $425 \mathrm{~nm}$. Addition of antioxidant sample to the "metHb-luminol- $\mathrm{H}_{2} \mathrm{O}_{2}$ " system leads to a change in the kinetics of chemiluminescence and prolongation of latent period $t$. There is a direct correlation of latent period and antioxidant activity of the added sample. The device "Lum-5373" was used to implement this method. The working volume of the mixture of "metHb- $\mathrm{H}_{2} \mathrm{O}_{2}$-luminol" with a buffer solution $(\mathrm{pH}=7.4)$ in the $\mathrm{CL}$ cell was $2.5 \mathrm{ml}$, and the sample injection volume of serum (v) varied from 1 to $20 \mu \mathrm{l}$. The latent period was calculated from the point of addition of hydrogen peroxide to the $\mathrm{CL}$ cell to the point of intersection of the tangent with the chemiluminescence time axis. The tangent of the line $(k$, $\left.\mu^{-1}\right)$ was taken as a criterion of antioxidant activity value of the samples, which describes the dependence between the relative latent period $\left(t / t_{0}\right)$ of $C L$ emission and the input sample volume to the cell $(v, \mu l): t / t_{0}=k v+1 ; t_{0}-$ latent period in the absence of the sample. Measurement error of this method based on the repeatability of results was $15 \%$.

\section{Voltammetric method (VA)}

The main principle of the voltammetric method for determining the total antioxidant activity in blood serum is the registration of the oxygen reduction in voltammograms with and without addition of the serum samples $(0.1 \mathrm{ml})$. The model reaction is the process of electroreduction of oxygen $\left(\mathrm{O}_{2} \mathrm{ER}\right)$, dissolved in a supporting electrolyte in a potential range of 0 to $-1.0 \mathrm{~V}$. This process is identical to the reduction of oxygen in living cells and tissues of the body.

In the VA method we used the coefficient of antioxidant activity (in $\mu \mathrm{mol} / / \times \mathrm{min}$ ) of the substances, which reflects the amount of active oxygen radicals having reacted with the antioxidants within a certain period of time, according to the formula:

$$
K=\left(1-\frac{l}{l_{0}}\right) \frac{C^{0}}{t},
$$

where $C^{0}[\mu \mathrm{mol} / \mathrm{l}]$ is the oxygen concentration in solution, $l$ is the ER $\mathrm{O}_{2}$ current with the addition of the investigated substance in the solution, $I_{0}$ is the limiting ER $\mathrm{O}_{2}$ current without the substance in the solution, and $t$ ( $\mathrm{min}$ ) is time of the interaction between the reactive oxygen species and an antioxidant at the working electrode. The electrochemical oxygen reduction at working mercury film electrode (MFE) progresses in several 
stages with the formation of reactive oxygen species (such as $\mathrm{O}_{2}^{\cdot-}$ and $\mathrm{HO}_{2}^{\cdot-}$ ), which interact with antioxidants at the electrode surface. The measurements were performed by the voltammetric analyzer "TA-2" (LLC “Tomanalyt” Tomsk, Russia) with an attached electrochemical cell consisting of a glassy carbon electrode indicator $\left(S=39 \mathrm{~mm}^{2}\right)$, silver chloride reference electrode and platinum wire auxiliary electrode. As the supporting electrolyte phosphate buffer $(\mathrm{pH}=6.86 ; 0.025 \mathrm{M}$ $\mathrm{KH}_{2} \mathrm{PO}_{4}, 0.025 \mathrm{M} \mathrm{Na}_{2} \mathrm{HPO}_{4}$ ) was used. The procedure for measuring the total antioxidant activity of blood serum was described previously [6]. The same method was applied to assess the content of thiol compounds in serum, also using this automated voltammetric analyzer "TA-2" [7]. The concentration of the total content of serum thiol compounds was determined by the method of the calibration chart by glutathione in the range of $1.0 \times 10^{-4}$ to $10.0 \times 10^{-4} \mathrm{~mol} / \mathrm{l}$ with the anodic peak height at the potential $\mathrm{E}=-0.04 \mathrm{~V}$.

\section{Amperometric method (AM)}

The amperometric approach of AOA evaluation is based on measurement of the electric current arising at oxidation of the investigated substance on the surface of a working electrode at a certain potential.

The amperometric flow-injection device "TsvetJauza-01-AA" used an electrochemical cell with a glassy-carbon anode and a stainless steel cathode to which a potential difference of $1.3 \mathrm{~V}$ was applied [8]. The analyte (pretreatment serum blood) was introduced into the eluent (solution of orthophosphoric acid with a concentration of $0.0022 \mathrm{~mol} / \mathrm{l}$ and a flow rate of $1.2 \mathrm{ml} / \mathrm{min}$ ); the eluent was driven through the electrochemical cell with a pump. The current of electrochemical oxidation was recorded versus the time, while the analyte passed through the cell. The integral signal (the surface area under a current curve in $\mathrm{nA} \times \mathrm{s})$ was compared to the signal received in the same conditions for the comparison sample with known concentration. The error in determination of the antioxidant content including the error by test preparation and reproducibility of results was within $10 \%$. Before the measurements, serum blood $(100 \mu \mathrm{l})$ was diluted with $900 \mu \mathrm{l}$ of distilled water. Then tubes with diluted serum were placed in a laboratory tabletop centrifuge and centrifuged for $10 \mathrm{~min}$ at a rotation speed of $2000 \mathrm{rpm}$. After that, $0.5 \mathrm{ml}$ of centrifugate was diluted with $50 \mathrm{ml}$ of water. The resulting solution was applied to the flow-injection system with the amperometric cell through a special six-way valve dispenser.

\section{Statistical analysis}

The means and standard errors of the mean were calculated. Statistical analysis was performed using Statistica 6.0 software. Student's $t$-test was used for statistical analysis of the data. The mean changes in coefficients of antioxidant activity of human serum were calculated for patients with alcoholism and healthy donors (control group). Data are presented as mean \pm SD.

\section{Results}

Investigation of the total antioxidant activity of blood serum by the voltammetric method revealed a decrease of the oxygen cathode current and shift of wave potential ER $\mathrm{O}_{2}$ towards positive values after addition of the blood samples in the background solution. That indicated the anti-

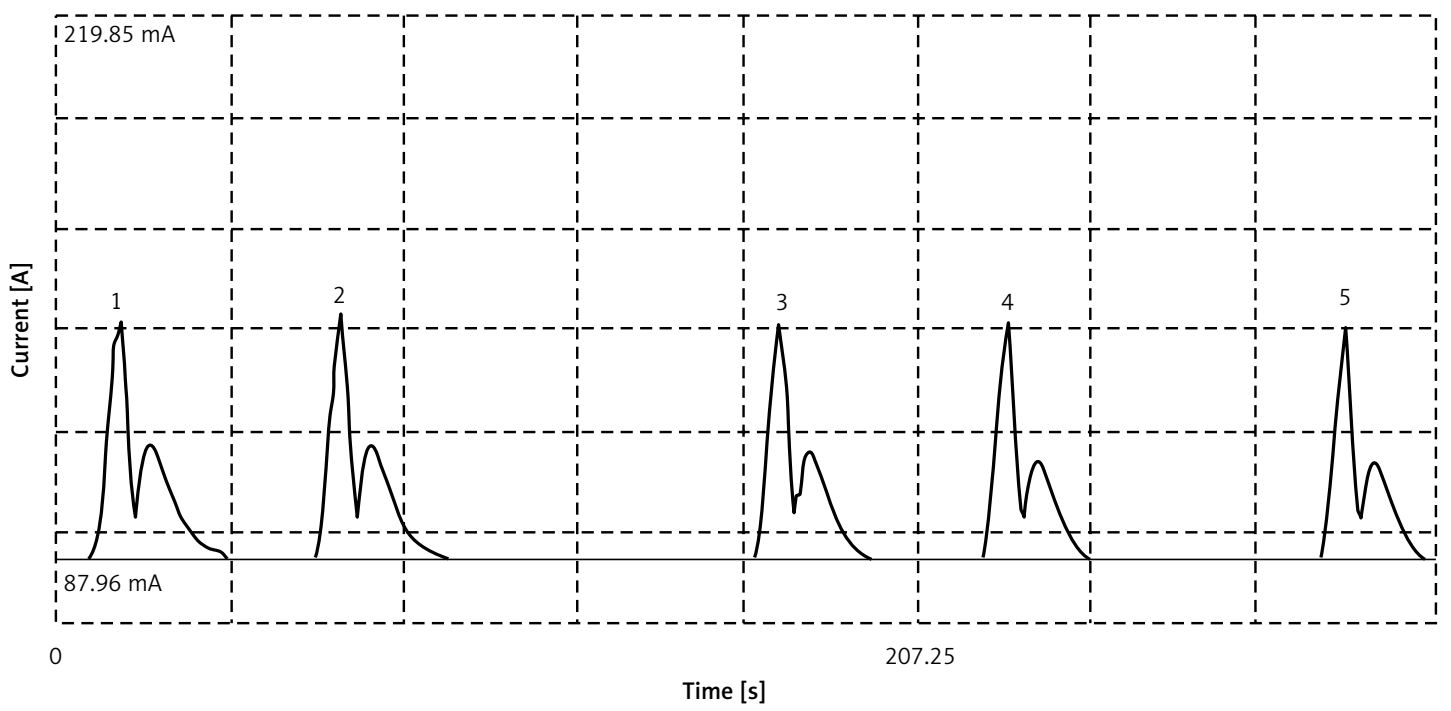

Figure 1. Amperogram of pretreatment serum samples with two-humped shape of current curves (by flow-injection analysis with amperometric detection) 
A

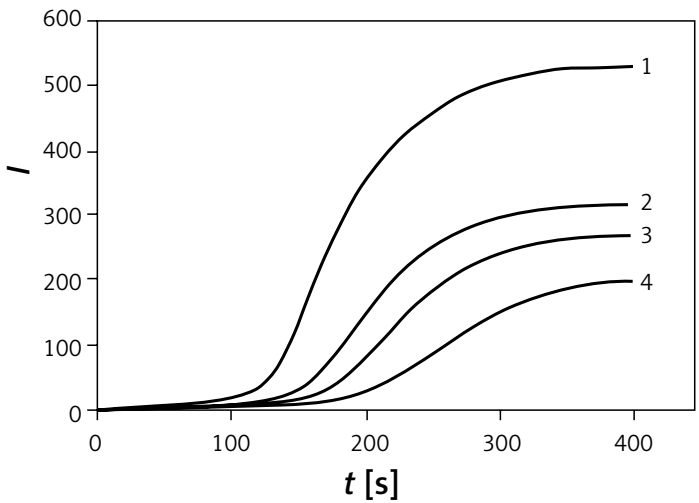

B

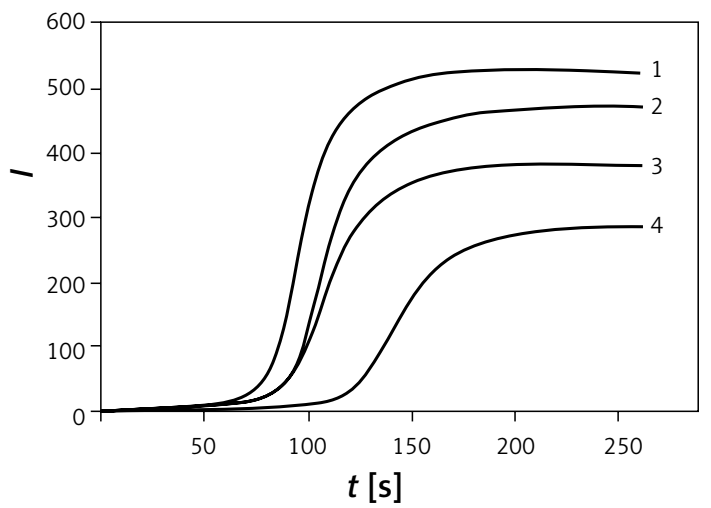

Figure 2. Intensity of CL emission (I) vs. time of process $(t)$. Dynamics of CL emission for different volumes of serum ((1) $-0 \mu \mathrm{l}$; (2) $-2 \mu \mathrm{l}$; (3) $-3 \mu \mathrm{l}$; (4) $-5 \mu \mathrm{l})$ of healthy donors (A) and alcoholics (B)

oxidant activity of the sample and allowed us to calculate the total AOA coefficient. The results of the blood serum test by the amperometric method are shown in Figure 1. This amperogram has an uncharacteristic two-humped shape, while for individual phenolic substances the one-humped form was always observed. This is probably due to the fact that oxidation of low-molecular components of blood serum associated with proteins provides a second peak due to the partial separation of the solution into various fractions on passing through the capillaries and amperometric cell.

A significant decrease of serum antioxidant activity of alcoholics compared with healthy donors was noted according to the $\mathrm{CL}$ method. Figure 2 shows the dynamics of luminescence for different sample volumes $(v, \mu l)$ of the donor $(A)$ and of the alcoholics (B) added into the chemiluminescence cell. The $\mathrm{CL}$ method revealed the biggest difference in TAA of serum between alcoholics and healthy donors (compared to other methods). The dependence between the relative latent period $\left(t / t_{0}\right)$ of $C L$ emission and the input sample volume to the cell $(v, \mu l)$ is well noted as a slope of the lines. It reflects TAA of the sample.

Low concentration of antioxidant in the blood of alcoholics leads a short latent period of chemiluminescence (Figure 2 B). Sera of healthy donors possess higher antioxidant capacity, which is manifested as a long latent period and the gradual slope of the intensity of chemiluminescence (Figure $2 \mathrm{~A}$ ).

The average values of serum antioxidant activity obtained by all used methods, are presented in Figure 3.

The applied methods are based on different principles and evaluate various aspects of the antioxidant properties of blood. The TAA is expressed in different units, as well as an additional parameter - the concentration of thiol compounds. However, the general trends of depletion of antioxidant properties in pathology are perfectly reflected by all methods.

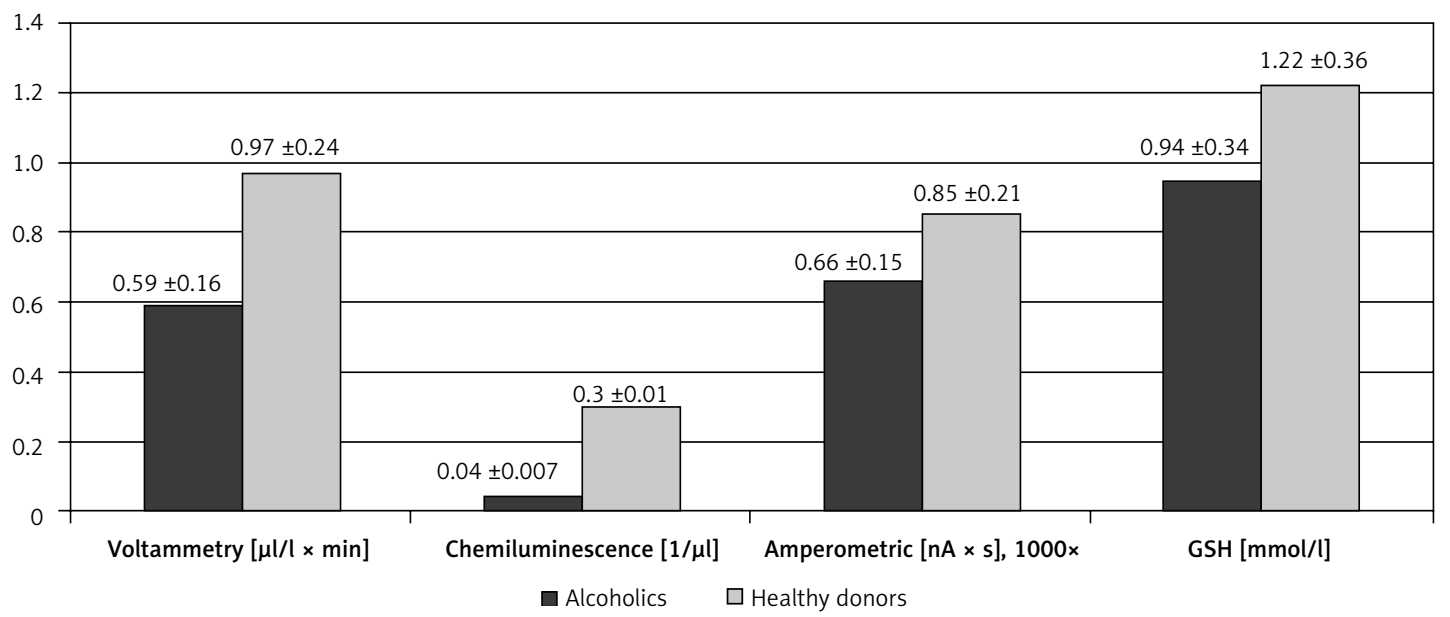

Figure 3. Average values (means \pm SD) of the total antioxidant activity and total content of thiol compounds in the blood serum of healthy donors and patients with a diagnosis of alcoholism according to VA, CL and AM methods $(p<0.05)$ 


\section{Discussion}

In our study, we observed changes in TAA level in the patients with alcoholism, which suggests that they were in a condition of oxidative stress. The results of the present study showed that oxidative stress may be one of the non-specific characteristic of alcoholism. The human organism contain many antioxidant enzyme systems and nonenzymatic antioxidants of different nature [9]. That is why it is quite difficult to compare directly, because these coefficients reflect different aspects of the antioxidant system. It should be noted that the criterion of TAA of blood serum reflects the interaction of antioxidants and certain radicals, depending on the model system that underlies a particular method. Another difficulty of TAA assessment is changes of the content of low molecular weight compounds in serum (mainly ascorbic acid and uric acid). It can vary significantly and can influence the results of electrochemical methods. A major role in whole antioxidant status may be played by the alimentary factor, determined by the patient's health condition and nutrition in the days preceding blood sampling. At the time of admission to hospital, patients are in a depleted state after a period of prolonged abuse of ethanol and often malnutrition. In our opinion, malnutrition can be a very important influence on TAA of blood. It should be noted for proper interpretation of the results of alcoholics. It partly explains observed discrepancies in the results of different methods. However, an equal general trend of the changes in serum antioxidant activity was revealed by all used methods. Coefficients of total antioxidant activity in blood serum of the patients with alcoholism were significantly lower than in the control group. This fact can be explained by alimentary insufficiency during the long period of severe alcohol intoxication of the patients. It is known that a simple regimen of antioxidant supplementation could be helpful in preventing formation of oxidative stress [10]. The dependence of the total antioxidant activity and thiol concentration in blood on the human health state is shown in Figure 3. Both parameters are lower in the case of alcoholism. The direct correlation between total antioxidant capacity (expressed as FRAP) and -SH group concentrations in the serum of patients was also reported [11]. Our study revealed that average concentration of thiols was $0.94 \pm 0.34 \mathrm{mmol} / \mathrm{l}$ ( $p<0.05)$ for patients with alcohol dependence, whereas it was $1.21 \pm 0.36 \mathrm{mmol} / /(p<0.05)$ for healthy donors. The decrease in thiol concentration in the group of patients with alcoholism may be explained by oxidative stress (confirmed by results of TAA assessment) and followed exhaustion of reduced thiols. This confirms that thiol compounds make a significant contribution to the an- tioxidant activity of serum. An important aspect is the ability of reduced glutathione (GSH) to reduce disulfide bonds of proteins and thus to restore the function of some enzymes. In this process, glutathione becomes the oxidized form (GSSG). Glutathione is also considered as a major endogenous antioxidant [12].

The results of determination of the total content of thiol compounds are in good agreement with the data on the evaluation of the total antioxidant activity of blood serum obtained by three independent methods (Figure 3). The review [13] reported similar changes in blood of alcoholics, including low levels of dietary antioxidant vitamins such as vitamin $C$ and vitamin $E$, and endogenous antioxidants such as blood glutathione. Reducing the concentration of thiols leads to an insufficient blood antioxidant system in alcoholics. Probably, such a condition could be corrected by special supplementation with antioxidants, as previously reported [14]. Antioxidant supplements can be considered as an essential component for complex alcoholism treatment.

In conclusion, the results of the experimental studies show that the proposed methods can be used for diagnostic purposes. Significant depletion of serum antioxidant activity in alcoholism was revealed by all methods. That may be a mark of alcohol-induced oxidative stress. Indicators of the total antioxidant activity of blood serum are largely dependent on the initial health state of the patient. However, for an objective assessment of antioxidant activity of biological objects it is advisable to use several analytical methods based on different model systems.

\section{Acknowledgments}

The study was supported by Russian Fund for Basic Research, research project No. 15-04-0111.

\section{Conflict of interest}

The authors declare no conflict of interest.

\section{References}

1. Korotkova El, Misini B, Dorozhko EV, Bukkel MV, Plotnikov EV, Linert W. Study of $\mathrm{OH}$ radicals in human serum blood of healthy individuals and those with pathological schizophrenia. Int J Mol Sci 2011; 12: 401-9.

2. Gumus S, Yucel O, Gamsizkan M, et al. The role of oxidative stress and effect of alpha-lipoic acid in reexpansion pulmonary edema - an experimental study. Arch Med Sci 2010; 6: 848-53.

3. Tomic S, Brkic S, Maric D, Mikic A. Lipid and protein oxidation in female patients with chronic fatigue syndrome. Arch Med Sci 2012; 8: 886-91.

4. Badarinath AV, Mallikarjuna $K$, Ao R, et al. A review on in-vitro antioxidant methods: comparisons, correlations and considerations. Intern J Pharm Tech Res 2010; 2: 1276-85. 
5. Teselkin JO, Babenkova IV, Lyubitsky OB, Klebanov GI, Vladimirov YA. Inhibition of luminol oxidation by serum antioxidants in presence of hydrogen peroxide and hemoglobin. Problems of Medical Chemistry (Rus) 1997; 43: 87-93.

6. Korotkova El, Freinbichler W, Linert W, et al. Study of total antioxidant activity of human serum blood in the pathology of alcoholism. Molecules 2013; 18: 1811-8.

7. Dorozhko EV, Korotkova El. Investigation of the electrochemical properties of glutathione by voltammetry. Proceedings of the higher educational institutions. Chemistry and Chemical Technology (Rus) 2010; 2: 35-8.

8. Yashin YI, Nemzer BV, Ryzhnev VY, Yashin AY, Chernousova NI, Fedina PA. Creation of a Databank for content of antioxidants in food products by an amperometric method. Molecules 2010; 15: 7450-66.

9. Sies H. Strategies of antioxidant defense. Eur J Biochem 1993; 215: 213-9.

10. Frei B, Stocker R, Ames BN. Antioxidant defenses and lipid peroxidation in human blood plasma. Proc Natl Acad Sci U S A 1988; 85: 9748-52.

11. Strzyżewski KW, Pioruńska-Stolzmann M, Majewski W, Kasprzak M, Strzyżewski M. Effect of surgical treatment on lipid peroxidation parameters and antioxidant status in the serum of ptients with peripheral arterial disease. Dis Markers 2013; 35: 647-52.

12. Percival M. Antioxidants. Clin Nutrition Insights NUT031 1/96 Rev. 10/98.

13. Kulkarni SR, Ravindra K, Dhume CY, Rataboli P, Rodrigue E. Levels of plasma testosterone, antioxidants and oxidative stress in alcoholic patients attending de-addiction centre. Biology and Medicine 2009; 1: 11-20.

14. Preobrazhensky S, Rabovsky A. Effects of broad-spectrum antioxidant supplementation on the antioxidant status of human plasma. 2000. USANA Clinical Research. Bulletin, USANA Health Sciences, Inc. SLC, UT. 